Article

\title{
Novel Guanidine Compound against Multidrug-Resistant Cystic Fibrosis-Associated Bacterial Species
}

\author{
Aamer Saeed 1,*(D), Alejandra Bosch ${ }^{2}$, Marisa Bettiol ${ }^{3}$, Diana L. Nossa González ${ }^{4}$, \\ Mauricio Federico Erben ${ }^{4, *}$ (D) and Yanina Lamberti ${ }^{2, *}$ \\ 1 Department of Chemistry, Quaid-I-Azam University, Islamabad 45320, Pakistan \\ 2 CINDEFI (UNLP, CONICET-CCT La Plata), Departamento de Química, Facultad de Ciencias Exactas, \\ Universidad Nacional de La Plata, La Plata 1900, Argentina; bosch@quimica.unlp.edu.ar \\ 3 Sala de Microbiología, Hospital de Niños Sor María Ludovica, La Plata 1900, Argentina; \\ microbiologialudovica@gmail.com \\ 4 CEQUINOR (UNLP, CONICET-CCT La Plata), Departamento de Química, Facultad de Ciencias Exactas, \\ Universidad Nacional de La Plata, La Plata 1900, Argentina; diana.nossa@uptc.edu.co \\ * Correspondence: asaeed@qau.edu.pk (A.S.); erben@quimica.unlp.edu.ar (M.F.E.); \\ ylamberti@quimica.unlp.edu.ar (Y.L.)
}

Received: 21 April 2018; Accepted: 8 May 2018; Published: 11 May 2018

\begin{abstract}
Chronic pulmonary infection is a hallmark of lung disease in cystic fibrosis (CF). Infections dominated by non-fermentative Gram-negative bacilli are particularly difficult to treat and highlight an urgent need for the development of new class of agents to combat these infections. In this work, a small library comprising thiourea and guanidine derivatives with low molecular weight was designed; these derivatives were studied as antimicrobial agents against Gram-positive, Gram-negative, and a panel of drug-resistant clinical isolates recovered from patients with CF. One novel compound, a guanidine derivative bearing adamantane-1-carbonyl and 2-bromo-4,6-difluouro-phenyl substituents (H-BDF), showed potent bactericidal activity against the strains tested, at levels generally higher than those exhibited by tobramycin, ceftazimide and meropenem. The role that different substituents exert in the antimicrobial activity has been determined, highlighting the importance of the halo-phenyl group in the guanidine moiety. The new compound displays low levels of cytotoxicity against THP- 1 and A549 cells with a selective index (SI) $>8$ (patent application PCT/IB2017/054870, August 2017). Taken together, our results indicate that H-BDF can be considered as a promising antimicrobial agent.
\end{abstract}

Keywords: antimicrobials; thioureas; guanidines; drug-resistant; cystic fibrosis

\section{Introduction}

In recent years, increasing infections due to antibiotic-resistant pathogens have made the formerly routine therapy of many infectious diseases challenging, and in many instances, extremely difficult or impossible to be eradicated [1-3]. Multidrug resistance is specially associated with respiratory tract infection in cystic fibrosis (CF) [4] where opportunistic pathogens such as Pseudomonas aeruginosa, Staphylococcus aureus, Stenotrophomonas maltophilia and species of the Burkholderia cepacia complex (Bcc) infect patient's lung and airways. Although for some patients the infection may occur only transiently, their acquisition most typically results in a chronic infection with acute debilitating exacerbations, causing a severe decline in respiratory function which contributes to disease progression and premature mortality $[5,6]$. In addition, they are important nosocomial pathogens affecting both immunocompetent and immunocompromised patients, and are responsible for a considerable proportion of infections in 
patients in Intensive Care Units (ICUs) worldwide [7]. Despite the emergence and dissemination of resistant bacteria and the need of more effective therapies, the development of new antimicrobial agents against these life-threatening infections is declining [8]. The impermeable nature of Gram-negative bacteria envelope, and the presence of multiple efflux pumps, in combination with other resistance mechanisms, has made the discovery of new effective antibacterial drugs very difficult [9].

Thioureas as well as guanidines represent two important groups of compounds due to their wide range of application as pharmaceutical agents. They possess a broad biological activity range including anti-inflammatory, anticancer, antiviral, antiparasitic, antifungal and antimicrobial properties $[10,11]$. Such a diverse range of biochemical behavior can be attributed to their flexible structure and the presence of nitrogen atoms in these molecules that make it possible to bear various substituents. For instance, it is well known that the 1-aroyl-3-(substituted-2-benzothiazolyl) thioureas exhibit potent antibacterial activity [12]. In addition, 1-(benzoyl)-3-(substituted) thioureas are antimicrobial agents [13] and the fluorinated analogues exhibit good antifungal activity [14]. Furthermore, due to efficient resonance stabilization of the charged protonated state, the guanidine groups have a relatively high acid dissociation constant which makes them stronger bases better suited for stable electrostatic interaction with the negative charged membranes of bacteria. This property improves the penetration of guanidine-bearing compounds through membranes and thus their biological activity $[15,16]$. On the other hand, the introduction of fluorine or appropriate fluorinated groups into organic compounds has advanced over recent decades in medicinal chemistry. The incorporation of fluorine atoms may contribute to increase metabolic stability, binding affinity and lipid solubility, thereby enhancing rates of absorption and transport of drugs in vivo $[17,18]$. Several studies further indicated that the incorporation of fluor and/or different electron withdrawing groups, such as bromo, chloro, acetyl, and nitro groups, on aromatic rings results in an improvement in antibacterial activity [16,19-21].

Taking into account the aforesaid biological and synthetic significance of thioureas and guanidines on one hand, and the multifunctional value of the electron withdrawing groups in drug design on the other, the endeavor of the current work was to investigate the activity of newly synthesized halophenyl substituted thioureas and guanidines against drug-resistant clinical isolates recovered from patients with CF.

\section{Materials and Methods}

\subsection{Reagents and Equipment}

1-adamantane carboxylic acid, thionyl chloride, triethylamine, potassium thiocyanate, mercury(II) chloride and substituted anilines were commercial products (Sigma-Aldrich, St. Louis, MO, USA) and were used as received. Analytical grade (Merck, Kenilworth, NJ, USA) acetone and dimethyl formamide, DMF, were dried and freshly distilled prior to use.

Melting points were recorded using a digital Gallenkamp (SANYO, Moriguchi, Japan) model MPD.BM 3.5 apparatus and are uncorrected. ${ }^{1} \mathrm{H}$ and ${ }^{13} \mathrm{C}$ NMR spectra were determined in $\mathrm{CDCl}_{3}$ at 300 $\mathrm{MHz}$ and 75.4 MHz, respectively, using a Bruker spectrophotometer (Billerica, Middlesex, MA, USA). FTIR spectra were acquired by a FTS $3000 \mathrm{MX}$ spectrometer. Elemental analyses were conducted using a LECO-183 CHNS analyzer (LECO Corporation, MI, USA). Thin layer chromatography (TLC) was carried out on 0.25 mm silica gel plates (60 F254, Merck, Darmstadt, Germany). Visualization was achieved by ultraviolet light.

\subsection{Synthesis of Compounds}

Thirteen compounds were synthesized and their structures were confirmed by a combination of elemental analysis, infrared and nuclear magnetic resonance spectroscopy. 1-(Adamantane-1-carbonyl)-3-substituted thiourea compounds were prepared by the addition reaction between adamantyl isothiocyanate with a variety of suitably substituted anilines [22-25]. The starting material 1-adamantane carbonyl chloride was obtained via the reaction of 1-adamantane carboxylic 
acid with thionyl chloride. A solution of adamantane-1-carbonyl chloride in dry acetone was treated with an equimolar quantity of potassium thiocyanate in dry acetone to yield the adamantane-1-carbonyl-isothiocyanate as intermediate (Figure 1). A treatment of the latter with an equimolar quantity of cyclohexylamine (for compound 1, Table 1) and a variety of substituted anilines (compounds 2-7, Table 1) in acetone produced the thiourea derivatives. In a typical procedure, a freshly distilled solution of adamantane-1-carbonyl chloride $(10 \mathrm{mmol})$ in dry acetone $(50 \mathrm{~mL})$ was added dropwise to a suspension of potassium thiocyanate $(10 \mathrm{mmol})$ in acetone $(30 \mathrm{~mL})$ and the reaction mixture was refluxed for $30 \mathrm{~min}$ under nitrogen. After cooling to room temperature, a solution of the substituted aniline $(10 \mathrm{mmol})$ in acetone $(10 \mathrm{~mL})$ was added and the resulting mixture refluxed for $2-4 \mathrm{~h}$. The reaction mixture was poured into cold water and the precipitated thioureas were recrystallized from suitable solvents.

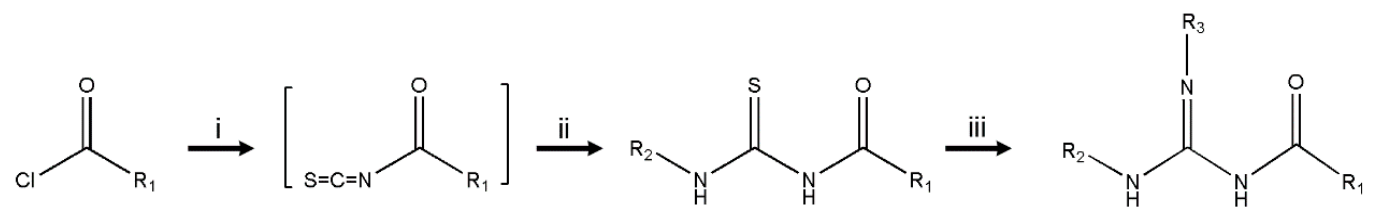

Figure 1. Synthesis of acyl thiourea and guanidine derivatives. Reagents and conditions: (i) Acyl chlorides and $\mathrm{KSCN}$ in dry acetone, $2 \mathrm{~h}$, reflux. (ii) Primary amines in dry acetone. (iii) $\mathrm{HgCl}_{2}$, substituted aniline and $\mathrm{Et}_{3} \mathrm{~N}$ in dry DMF.

Three 1-acyl-3-(2-bromo-4,6-difluoro-phenyl)thioureas (compounds 8-10, Table 1) were synthesized in a similar way by treating the corresponding acyl chloride derivatives (1-naphthoyl chloride, 2,4-dichloro-benzoyl chloride and 4-methyl-benzoyl chloride, respectively) with potassium thiocyanate in dry acetone followed by the addition of 2-bromo-4,6-difluoro-aniline.

For the synthesis of guanidine derivatives (compounds 11-13, Figure 2), the general method proposed by Vencato and coworkers [26] was applied (Figure 1). In a typical procedure triethylamine $(2.8 \mathrm{~mL}, 20 \mathrm{mmol})$ and selected anilines $(10 \mathrm{mmol})$ were added successively to a stirred solution of the corresponding 1-(adamantane-1-carbonyl)thiourea $(10 \mathrm{mmol})$ in $\mathrm{DMF}(20 \mathrm{~mL})$ at $10^{\circ} \mathrm{C}$ followed by the addition of mercury(II) chloride $(2.72 \mathrm{~g}, 10 \mathrm{mmol})$. The reaction mixture was stirred at room temperature for $12 \mathrm{~h}$ and then filtered to remove the $\mathrm{HgS}$. The filtrate was extracted with $\mathrm{EtOAc} / \mathrm{H}_{2} \mathrm{O}$ $(1: 1)(3 \times 5 \mathrm{~mL})$, the organic phase dried over anhydrous $\mathrm{Na}_{2} \mathrm{SO}_{4}$, and concentrated in vacuum to leave an oily residue which recrystallized on standing.

1-(Adamantane-1-carbonyl)-3-cyclohexylthiourea (1). Yield 68\%, semisolid; FT-IR $\left(\mathrm{KBr}, \mathrm{v}, \mathrm{cm}^{-1}\right)$ : $3336(\mathrm{NH}), 3034(\mathrm{Ar}-\mathrm{CH}), 2926\left(\mathrm{CH}_{2}\right), 2909,2849\left(\mathrm{CH}_{2}, \mathrm{CH}\right), 1675(\mathrm{C}=\mathrm{O}), 1575$, 1457, $1370(\mathrm{C}=\mathrm{S})$. ${ }^{1} \mathrm{H}$ NMR (300 MHz, CDCl $)_{3}$ ): $\delta 13.08$ (br s, $1 \mathrm{H}, \mathrm{NH}, \mathrm{D}_{2} \mathrm{O}$ exchangeable); $6.25(1 \mathrm{H}, \mathrm{s}$, broad, $\mathrm{NH}$ ); 4.09 (br s, $1 \mathrm{H}, \mathrm{NH}, \mathrm{D}_{2} \mathrm{O}$ exchangeable); $3.94(1 \mathrm{H}, \mathrm{m}, \mathrm{CH}), 2.1$ (br s, 3H, adamantane- $\left.\mathrm{CH}\right), 1.95(\mathrm{~s}, 6 \mathrm{H}$, adamantane- $\left.\mathrm{CH}_{2}\right), 1.94-2.02\left(2 \mathrm{H}, \mathrm{dd}, \mathrm{CH}_{2}\right), 1.60-1.76\left(4 \mathrm{H}, \mathrm{m}, \mathrm{CH}_{2} \times 2\right), 1.79\left(\mathrm{~m}, 6 \mathrm{H}\right.$, adamantane- $\left.\mathrm{CH}_{2}\right)$ 1.18-1.45 (4H, m, $\left.\mathrm{CH}_{2} \times 2\right) ;{ }^{13} \mathrm{C} \mathrm{NMR}\left(75 \mathrm{MHz}, \mathrm{CDCl}_{3}\right)$ : $179.1(\mathrm{C}=\mathrm{S}) ; 178.46(\mathrm{C}=\mathrm{O}), 54.37(\mathrm{CH}), 41.98$, 41.90, 39.2, 38.5, 36.4, 36.0, 33.03 $\left(\mathrm{CH}_{2}-4\right), 32.81\left(\mathrm{CH}_{2}-2\right), 31.6$, 28.0, $24.75\left(\mathrm{CH}_{2}-3\right), 27.7$, (adamantane-C) $25.41\left(\mathrm{CH}_{2}-3\right)$, $24.75\left(\mathrm{CH}_{2}-3\right)$; Anal. Calcd for $\mathrm{C}_{18} \mathrm{H}_{28} \mathrm{~N}_{2} \mathrm{OS}$ (320.19): $\mathrm{C}, 67.46 ; \mathrm{H}, 8.81 ; \mathrm{N}, 8.74 ; \mathrm{S}, 10.00 \%$; Found: $\mathrm{C}, 67.46 ; \mathrm{H}, 8.81 ; \mathrm{N}, 8.74 ; \mathrm{S}, 10.00 \%$.

1-(Adamantane-1-carbonyl)-3-phenylthiourea (2). Yield 72\%, mp 108-110 ${ }^{\circ} \mathrm{C}$. FT-IR $\left(\mathrm{KBr}, \mathrm{v}, \mathrm{cm}^{-1}\right)$ : $3336(\mathrm{NH}), 3034(\mathrm{Ar}-\mathrm{CH}), 2909,2849\left(\mathrm{CH}_{2}, \mathrm{CH}\right), 1679$ (C=O), 1575, 1457, 1375 (C=S); ${ }^{1} \mathrm{H}$ NMR $(300 \mathrm{MHz}$, $\mathrm{CDCl}_{3}$ ): $\delta 12.71$ (br s, $1 \mathrm{H}, \mathrm{NH}, \mathrm{D}_{2} \mathrm{O}$ exchangeable); 7.63 (br s, $1 \mathrm{H}, \mathrm{NH}, \mathrm{D}_{2} \mathrm{O}$ exchangeable); 7.23-7.33 $(\mathrm{m}, 2 \mathrm{H}, \mathrm{Ar}) ; 7.38-7.43(\mathrm{~m}, 2 \mathrm{H}, \mathrm{Ar}), 8.40-8.48(\mathrm{~m}, 1 \mathrm{H}, \mathrm{Ar}) ; 2.08(\mathrm{~s}, 3 \mathrm{H}$, adamantane-CH), $1.69(\mathrm{~s}, 6 \mathrm{H}$, adamantane- $\left.\mathrm{CH}_{2}\right), 1.58\left(\mathrm{q}, 6 \mathrm{H}\right.$, adamantane- $\left.\mathrm{CH}_{2}, J=8.6 \mathrm{~Hz}\right) ;{ }^{13} \mathrm{C} \mathrm{NMR}\left(75 \mathrm{MHz}, \mathrm{CDCl}_{3}\right)$ : $179.6(\mathrm{C}=\mathrm{S})$; $170.12(\mathrm{C}=\mathrm{O})$; 143.05 (C-9); 41.51, 39.25, 38.69, 38.49, 36.44, 36.14, 28.05, 27.86, 27.78, (adamantane-C); Anal. Calcd for $\mathrm{C}_{18} \mathrm{H}_{22} \mathrm{~N}_{2} \mathrm{OS}$ (314.45): C, 68.75; H, 7.05; N, 8.91; S, 10.20\%; Found: C, 68.83; H, 7.10; N, 8.98; S, 10.14\%. 
1-(Adamantane-1-carbonyl)-3-(4-methyl-3-fluorophenyl)thiourea (3). Yield 69\%, mp 174-176 ${ }^{\circ} \mathrm{C}$. FT-IR $\left(v, \mathrm{~cm}^{-1}\right): 3436,3034,2909,1675,1585,1457,1368 .{ }^{1} \mathrm{H}$ NMR $\left(300 \mathrm{MHz}, \mathrm{CDCl}_{3}\right): \delta 12.47$ (br s, 1H, NH, D $2 \mathrm{O}$ exchangeable); 8.53 (br s, 1H, NH, $\mathrm{D}_{2} \mathrm{O}$ exchangeable); 7.19 (s, 1H, Ar), 7.59 (s, $1 \mathrm{H}, \mathrm{Ar}), 7.81(\mathrm{~d}, 2 \mathrm{H}, \mathrm{J}=8.6 \mathrm{~Hz}, \mathrm{Ar}), 2.37$ (s, 3H, Ar-CH $) 2.14$ (brs, 3H, adamantane-CH), 1.95 (s, $6 \mathrm{H}$, adamantane- $\left.\mathrm{CH}_{2}\right), 1.79\left(\mathrm{q}, 6 \mathrm{H}\right.$, adamantane- $\left.\mathrm{CH}_{2}, J=8.6 \mathrm{~Hz}\right) ;{ }^{13} \mathrm{C} \mathrm{NMR}\left(75 \mathrm{MHz}, \mathrm{CDCl}_{3}\right): 178.9(\mathrm{C}=\mathrm{S})$, 177.1 (C=O), 161.7 (Ar), 136.7 (Ar), 135.1 (Ar), 136.7 (Ar), 129.7, 141.4, 124.2 (ArCs), $21.2\left(\mathrm{Ar}-\mathrm{CH}_{3}\right)$ 38.44, 36.14, 27.86, 21.78, (adamantane-C); Anal. Calcd for $\mathrm{C}_{19} \mathrm{H}_{23} \mathrm{FN}_{2} \mathrm{OS}$ (346.15): C, 65.87; H, 6.69; N, 8.09; S, 9.25\%; Found: C, 65.739; H, 6.72; N, 7.97; S, 9.23\%.

1-(Adamantane-1-carbonyl)-3-(2-nitrophenyl)thiourea (4). Yield 73\%, mp 160-162 ${ }^{\circ} \mathrm{C}$. FT-IR (KBr, $\left.v, \mathrm{~cm}^{-1}\right): 3336(\mathrm{NH}), 3034(\mathrm{Ar}-\mathrm{CH}), 2909,2849\left(\mathrm{CH}_{2}, \mathrm{CH}\right), 1682(\mathrm{C}=\mathrm{O}), 1586,1543\left(\mathrm{NO}_{2}\right.$ asymmetric $)$ 1457, 1368 (C=S), $1340 \mathrm{~cm}^{-1}\left(\mathrm{NO}_{2}\right.$ symmetric); ${ }^{1} \mathrm{H}$ NMR (300 MHz, CDCl $): \delta 12.71$ (br s, $1 \mathrm{H}, \mathrm{NH}, \mathrm{D}_{2} \mathrm{O}$ exchangeable); 7.63 (br s, $1 \mathrm{H}, \mathrm{NH}, \mathrm{D}_{2} \mathrm{O}$ exchangeable); 7.23-7.33 (m, 2H, Ar); 7.38-7.43 (m, 2H, Ar); 2.08 (s, 3H, adamantane-CH), $1.69\left(\mathrm{~s}, 6 \mathrm{H}\right.$, adamantane- $\left.\mathrm{CH}_{2}\right), 1.58\left(\mathrm{q}, 6 \mathrm{H}\right.$, adamantane- $\left.\mathrm{CH}_{2}, J=8.6 \mathrm{~Hz}\right)$; ${ }^{13} \mathrm{C}$ NMR (75 MHz, $\left.\mathrm{CDCl}_{3}\right)$ : $179.6(\mathrm{C}=\mathrm{S}) ; 170.12$ (C=O); 143.05 (C-9); 41.51, 39.25, 38.69, 38.49, 36.44, 36.14, 28.05, 27.86, 27.78, (adamantane-C); Anal. Calcd for $\mathrm{C}_{18} \mathrm{H}_{21} \mathrm{~N}_{3} \mathrm{O}_{3} \mathrm{~S}$ (359.44): C, 60.15; $\mathrm{H}, 5.89$; N, 11.69; O, 13.35; S, 8.92\%; Found: C, 60.21; H, 5.93; N, 11.71; S, 8.89\%.

1-(Adamantane-1-carbonyl)-3-(4-acetyl-phenyl)thiourea (5). Yield 160-161 ${ }^{\circ} \mathrm{C}$. FT-IR (KBr, v, $\left.\mathrm{cm}^{-1}\right)$ : 3336 (NH), 3034 (Ar-CH), 2909, 2849 ( $\left.\mathrm{CH}_{2}, \mathrm{CH}\right), 1679$ (C=O), 1575, 1457, 1375 (C=S). ${ }^{1} \mathrm{H} \mathrm{NMR}(300 \mathrm{MHz}$, $\mathrm{CDCl}_{3}$ ): $\delta 12.74$ (br s, $1 \mathrm{H}, \mathrm{NH}, \mathrm{D}_{2} \mathrm{O}$ exchangeable), 9.83 (br s, 1H, NH, $\mathrm{D}_{2} \mathrm{O}$ exchangeable), 7.91 (d, 2H, $J=8.6 \mathrm{~Hz}, \mathrm{Ar}) ; 7.73(\mathrm{~d}, 2 \mathrm{H}, J=8.6 \mathrm{~Hz}, \mathrm{Ar}), 2.3\left(\mathrm{~s}, 3 \mathrm{H}, \mathrm{CH}_{3} \mathrm{CO}\right), 2.08$ (s, 3H, adamantane-CH), $1.69(\mathrm{~s}, 6 \mathrm{H}$, adamantane- $\left.\mathrm{CH}_{2}\right), 1.58\left(\mathrm{q}, 6 \mathrm{H}\right.$, adamantane- $\left.\mathrm{CH}_{2}, J=8.6 \mathrm{~Hz}\right) ;{ }^{13} \mathrm{C} \mathrm{NMR}\left(75 \mathrm{MHz}, \mathrm{CDCl}_{3}\right): \delta 193.6(\mathrm{CO})$, $179.6(\mathrm{C}=\mathrm{S}), 174.5(\mathrm{C}=\mathrm{O}), 143.0,138.0,132.6,127.8,28.1\left(\mathrm{CH}_{3}\right), 41.51,39.25,38.69,38.49,36.44,36.14$, 28.05, 27.86, 27.78, (adamantane-C); Anal. Calcd for $\mathrm{C}_{20} \mathrm{H}_{24} \mathrm{~N}_{2} \mathrm{O}_{2} \mathrm{~S}$ (356.47): C, 67.39; $\mathrm{H}, 6.79 ; \mathrm{N}, 7.86$; S, 8.99\%; Found: C, 67.42; H, 6.83; N, 7.81; S, 8.91\%.

1-(Adamantane-1-carbonyl)-3-(2,3-dichlorophenyl)thiourea (6). Yield 79\%, mp 196-198 ${ }^{\circ} \mathrm{C}$. FT-IR (KBr, v, cm $\left.{ }^{-1}\right)$ : 3336 (NH), 3034 (Ar-CH), 2909, 2849 ( $\left.\mathrm{CH}_{2}, \mathrm{CH}\right), 1675$ (C=O), 1575, 1457, 1370 (C=S). ${ }^{1} \mathrm{H}$ NMR (300 MHz, CDCl 3 ): $\delta 12.74$ (br s, 1H, NH, D $2 \mathrm{O}$ exchangeable); 8.70 (br s, 1H, NH, D exchangeable); $8.03(\mathrm{~d}, 1 \mathrm{H}, J=8.6 \mathrm{~Hz} \mathrm{Ar}), 7.96(\mathrm{~d}, 1 \mathrm{H}, J=8.6 \mathrm{~Hz} \mathrm{Ar}), 7.90(\mathrm{~d}, 1 \mathrm{H}, J=8.6 \mathrm{~Hz} \mathrm{Ar}), 7.83(\mathrm{~d}$, $1 \mathrm{H}, J=8.6 \mathrm{~Hz} \mathrm{Ar}), 7.57$ (m, 3H, Ar), 2.1 (br s, 3H, adamantane-CH), 2.03 (s, 6H, adamantane-CH $\mathrm{CH}_{2}$, 1.81 (q, 6H, adamantane-CH,$J=8.6 \mathrm{~Hz}) ;{ }^{13} \mathrm{C} \mathrm{NMR}\left(75 \mathrm{MHz}_{2} \mathrm{CDCl}_{3}\right): 178.9(\mathrm{C}=\mathrm{S}) ; 134.10(\mathrm{Ar}), 128.6$, 126.9 125.3,123.64, 121.67 (ArCs), 41.94, 41.90, 39.2, 38.6, 36.1, 36.0, 31.6, 28.0, 27.8, (adamantane-C); Anal. Calcd for $\mathrm{C}_{18} \mathrm{H}_{2 \mathrm{o}} \mathrm{Cl}_{2} \mathrm{~N}_{2} \mathrm{OS}$ (383.34): C, 56.40; H, 5.26; N, 7.31; S, 8.36\%; Found: C, 56.40; H, 5.26; $\mathrm{N}, 7.31 ; \mathrm{S}, 8.36 \%$.

1-(Adamantane-1-carbonyl)-3-(2-bromo-4,6-difluorophenyl)thiourea (7). Yield 70\%, mp 194-196 ${ }^{\circ} \mathrm{C}$. FT-IR (KBr, v, cm $\left.{ }^{-1}\right)$ : 3336 (NH), 3034 (Ar-CH), 2909, $2849\left(\mathrm{CH}_{2}, \mathrm{CH}\right), 1675$ (C=O), 1575, 1457, 1370 (C=S). ${ }^{1} \mathrm{H}$ NMR (300 MHz, CDCl $): \delta 11.93$ (br s, 1H, NH, D $2 \mathrm{O}$ exchangeable), 9.61 (br s, 1H, NH, $\mathrm{D}_{2} \mathrm{O}$ exchangeable), 7.48-7.44 (m, 1H, Ar), 7.29-7.22 (m, 1H, Ar), $2.08(\mathrm{t}, 10 \mathrm{H}$, adamantane-H, $J=6.0 \mathrm{~Hz})$, $1.80(\mathrm{t}, 6 \mathrm{H}$, adamantane-H, $J=4.8 \mathrm{~Hz}) ;{ }^{13} \mathrm{C} \mathrm{NMR}\left(75 \mathrm{MHz}, \mathrm{CDCl}_{3}\right): 182.3(\mathrm{C}=\mathrm{S}), 179.2(\mathrm{C}=\mathrm{O}), 163.3$, 160.6, 159.8, 157.2, 123.9, 115.8, 104.5, 103.8 (ArCs), 41.9, 37.6, 35.8, (adamantane-C); Anal. Calcd for $\mathrm{C}_{18} \mathrm{H}_{19} \mathrm{~F}_{2} \mathrm{BrN}_{2} \mathrm{OS}$ (429.32): C, 50.36; H, 4.46; N, 6.53; S, 7.47; Found: C, 50.24; H, 4.51; N, 6.57; S, 7.36\%.

1-(1-naphtyl)-3-(2-bromo-4,6-difluoro-phenyl)thiourea (8). Yield 81\%, mp 174-176 ${ }^{\circ} \mathrm{C}$. FT-IR (KBr, $\left.v, \mathrm{~cm}^{-1}\right): 3336(\mathrm{NH}), 3034(\mathrm{Ar}-\mathrm{CH}), 1671(\mathrm{C}=\mathrm{O}), 1585,1451,1372(\mathrm{C}=\mathrm{S}) .{ }^{1} \mathrm{H} \mathrm{NMR}\left(300 \mathrm{MHz}, \mathrm{CDCl}_{3}\right)$ : $\delta 11.98$ (br s, 1H, NH, $\mathrm{D}_{2} \mathrm{O}$ exchangeable); 11.29 (br s, 1H, NH, D $2 \mathrm{O}$ exchangeable); 8.89-6.71 (m, 9H, Ar); ${ }^{13} \mathrm{C}$ NMR (75 MHz, $\left.\mathrm{CDCl}_{3}\right): 168.9,164.3,145.1,132.0,134,120.4,125.1,129.6,116,103.1$ (ArCs); Anal. Calcd for $\mathrm{C}_{18} \mathrm{H}_{11} \mathrm{~F}_{2} \mathrm{BrN}_{2} \mathrm{OS}$ (421.97): C, 51.32; H, 2.63; N, 6.65 S, 7.61\%; Found: C, 51.24; H, 2.60; $\mathrm{N}, \mathrm{S}, 6.61,7.57 \%$.

1-(2,4-dichloro-phenyl)-3-(2-bromo-4,6-difluoro-phenyl)thiourea (9). Yield 81\%, mp 174-176 ${ }^{\circ} \mathrm{C}$. FT-IR (KBr, v, cm $\left.{ }^{-1}\right)$ : $3336(\mathrm{NH}), 3034$ (Ar-CH), 2909, $2849\left(\mathrm{CH}_{2}, \mathrm{CH}\right), 1675$ (C=O), 1575, 1457, 
1370 (C=S). ${ }^{1} \mathrm{H}$ NMR (300 MHz, $\mathrm{CDCl}_{3}$ ): $\delta 12.07$ (br s, $1 \mathrm{H}, \mathrm{NH}, \mathrm{D}_{2} \mathrm{O}$ exchangeable); 11.35 (br s, $1 \mathrm{H}$, $\mathrm{NH}, \mathrm{D}_{2} \mathrm{O}$ exchangeable); $7.61(\mathrm{~s}, 1 \mathrm{H}, \mathrm{Ar}), 7.58(\mathrm{~d}, 1 \mathrm{H}, J=8.3 \mathrm{~Hz}, \mathrm{Ar}), 7.58(\mathrm{~d}, 1 \mathrm{H}, J=8.3 \mathrm{~Hz}, \mathrm{Ar}), 7.49(\mathrm{~s}$, 1H, Ar), 7.19 (s, 1H, Ar); $\left.{ }^{13} \mathrm{C} \mathrm{NMR} \mathrm{(75} \mathrm{MHz,} \mathrm{CDCl}\right)$ : 181.9 (C=S); 170.3 (C=O), 168.2, 159.5, 134.10 (Ar), 141.4, 134.1, 130.1, 129.4, 127.3, 128.6, 126.9, 124.2, 119.7, 114.9 (ArCs); Anal. Calcd for $\mathrm{C}_{14} \mathrm{H}_{17} \mathrm{Cl}_{2}$ $\mathrm{F}_{2} \mathrm{BrN}_{2} \mathrm{OS}$ (439.88): C, 38.21; H, 1.60; N, 6.37; S, 7.28\%; Found: C, 37.28; H, 1.62; N, 6.33; S, 8.30\%.

1-(4-methylphenyl)-3-(2-bromo-4,6-difluoro-phenyl)thiourea (10). Yield 69\%, mp 174-176 ${ }^{\circ} \mathrm{C}$. FT-IR $\left(\mathrm{KBr}, \mathrm{v}, \mathrm{cm}^{-1}\right)$ : $3436(\mathrm{NH}), 3034(\mathrm{Ar}-\mathrm{CH}), 2909,1675$ (C=O), 1585, 1457, 1368 (C=S). ${ }^{1} \mathrm{H}$ NMR (300 MHz, $\mathrm{CDCl}_{3}$ ): $\delta 12.74$ (br s, $1 \mathrm{H}, \mathrm{NH}, \mathrm{D}_{2} \mathrm{O}$ exchangeable); 11.31 (br s, $1 \mathrm{H}, \mathrm{NH}, \mathrm{D}_{2} \mathrm{O}$ exchangeable); 7.19 (s, $1 \mathrm{H}, \mathrm{Ar}), 7.59(\mathrm{~s}, 1 \mathrm{H}, \mathrm{Ar}), 7.81(\mathrm{~d}, 2 \mathrm{H}, J=8.6 \mathrm{~Hz}, \mathrm{Ar}), 2.51\left(\mathrm{~s}, 3 \mathrm{H}, \mathrm{Ar}_{-} \mathrm{CH}_{3}\right) ;{ }^{13} \mathrm{C} \mathrm{NMR}\left(75 \mathrm{MHz}, \mathrm{CDCl}_{3}\right)$ : $178.9(\mathrm{C}=\mathrm{S}), 173.1(\mathrm{C}=\mathrm{O}), 134.10(\mathrm{Ar}), 181.7,141.4,130.1,128.6,126.9,124.2,119.7,114.9$ (ArCs), 19.4 (Ar- $\mathrm{CH}_{3}$ ); Anal. Calcd for $\mathrm{C}_{15} \mathrm{H}_{11} \mathrm{~F}_{2} \mathrm{BrN}_{2} \mathrm{OS}$ (385.97): C, 46.77; $\mathrm{H}, 2.88 ; \mathrm{N}, 7.27 ; \mathrm{S}, 8.32 \%$; Found: $\mathrm{C}$, $46.81 ; \mathrm{H}, 2.92 ; \mathrm{N}, 7.23 ; \mathrm{S}, 8.28 \%$.

1-(Adamantane-1-carbonyl)-2,3-bis(2-bromo-4,6-difluoro-phenyl)guanidine (11). Yield 70\%, mp 148-149 ${ }^{\circ}$ C. FT-IR (KBr, v, cm ${ }^{-1}$ ): 3336, 3413, 3245, 3128, 3043, 3034, 2909, 2849, 1675, 1575, 1457, 1370. ${ }^{1} \mathrm{H}$ NMR (300 MHz, CDCl $)$ ): $\delta 9.79$ (br s, $1 \mathrm{H}, \mathrm{NH}, \mathrm{D}_{2} \mathrm{O}$ exchangeable); 8.04 (br s, $1 \mathrm{H}, \mathrm{NH}, \mathrm{D}_{2} \mathrm{O}$ exchangeable); 7.17-7.13 (m, 2H, Ar), 7.06-6.98 (m, 2H, Ar), 2.0 (br s, 3H, adamantane-H), 1.94-1.89 (br m, 3H, adamantane-H), 1.78-1.60 (br m, 10H, adamantane-H); ${ }^{13} \mathrm{C}$ NMR $\left(75 \mathrm{MHz}, \mathrm{CDCl}_{3}\right): 178.2$ $(\mathrm{C}=\mathrm{O}), 174.2(\mathrm{C}=\mathrm{N}), 154.9,151.8,148.6,131.9,114.6,107.8,103.2(\mathrm{ArCs}), 40.9,37.9,35.8$, (adamantane-C); Anal. Calcd for $\mathrm{C}_{24} \mathrm{H}_{21} \mathrm{~F}_{4} \mathrm{Br}_{2} \mathrm{~N}_{3} \mathrm{O}$ (603.2): C, 47.78; H, 3.51; N, 6.97\%; Found: $\mathrm{C}, 48.1 ; \mathrm{H}, 3.49 ; \mathrm{N}, 7.01 \%$.

\section{1-(Adamantane-1-carbonyl)-2-(2-bromo-4,6-difluoro-phenyl)-3(2,6-di-bromo-4-fluoro-phenyl)} guanidine (12). Yield $70 \%$, mp $144-145^{\circ} \mathrm{C}$. FT-IR $\left(\mathrm{KBr}, v, \mathrm{~cm}^{-1}\right)$ : 3413, 3245, 3128, 3043, 3034, 2909, 2849, 1675, 1575, 1457, 1370. ${ }^{1} \mathrm{H}$ NMR (300 MHz, CDCl $): \delta 11.94$ (br s, $1 \mathrm{H}, \mathrm{NH}, \mathrm{D}_{2} \mathrm{O}$ exchangeable); 9.66 (br s, $1 \mathrm{H}, \mathrm{NH}, \mathrm{D}_{2} \mathrm{O}$ exchangeable); $7.48(\mathrm{~m}, 1 \mathrm{H}, \mathrm{Ar}), 7.23(\mathrm{~m}, 2 \mathrm{H}, \mathrm{Ar}), 7.01(\mathrm{~m}, 1 \mathrm{H}, \mathrm{Ar}), 1.99-1.84$ (m, 10H, adamantane-H), 1.79-1.59 (m, 6H, adamantane-H); ${ }^{13} \mathrm{C}$ NMR $\left(75 \mathrm{MHz}, \mathrm{CDCl}_{3}\right): 179.2(\mathrm{C}=\mathrm{O})$, $174.2(\mathrm{C}=\mathrm{N}), 160.4,159.8,157.2,151.9,147.2,140.2,123.9,115.8,114.3,104.5$ (ArCs), 41.9, 37.6, 35.8 (adamantane-C); Anal. Calcd for $\mathrm{C}_{24} \mathrm{H}_{21} \mathrm{~F}_{3} \mathrm{Br}_{3} \mathrm{~N}_{3} \mathrm{O}$ (664.2): C, 43.40; H, 3.19; N, 6.33\%; Found: C, 43.21.1; H, 3.52; N, 6.97\%.

1-(Adamantane-1-carbonyl)-2,3-bis-(2-nitro-phenyl)guanidine (13). Yield 70\%, mp $156{ }^{\circ} \mathrm{C}$. FT-IR $\left(\mathrm{KBr}, v, \mathrm{~cm}^{-1}\right)$ : 3336, 3413, 3245, 3128, 3043, 3034, 2909, 2849, 1675, 1575, 1457, 1370. ${ }^{1} \mathrm{H}$ NMR $(300 \mathrm{MHz}$, $\mathrm{CDCl}_{3}$ ): $\delta 11.94$ (br s, $1 \mathrm{H}, \mathrm{NH}, \mathrm{D}_{2} \mathrm{O}$ exchangeable); 9.66 (br s, $1 \mathrm{H}, \mathrm{NH}, \mathrm{D}_{2} \mathrm{O}$ exchangeable); 7.48 (m, $1 \mathrm{H}, \mathrm{Ar}), 7.23(\mathrm{~m}, 2 \mathrm{H}, \mathrm{Ar}), 7.01(\mathrm{~m}, 1 \mathrm{H}, \mathrm{Ar}), 1.99-1.84(\mathrm{~m}, 10 \mathrm{H}$, adamantane-H), 1.79-1.59 (m, 6H, adamantane-H); ${ }^{13} \mathrm{C}$ NMR $\left(75 \mathrm{MHz}, \mathrm{CDCl}_{3}\right)$ : $179.2(\mathrm{C}=\mathrm{O}), 174.2(\mathrm{C}=\mathrm{N}), 160.4,159.8,157.2,151.9,147.2$, 140.2, 123.9, 115.8, 114.3, 104.5 (ArCs), 41.9, 37.6, 35.8 (adamantane-C); Anal. Calcd for $\mathrm{C}_{24} \mathrm{H}_{25} \mathrm{~N}_{5} \mathrm{O}_{5}$ (463.5): C, 62.19; H, 5.44; N, 15.11\%; Found: C, 61.97.1; H, 5.42; N, 6.93\%.

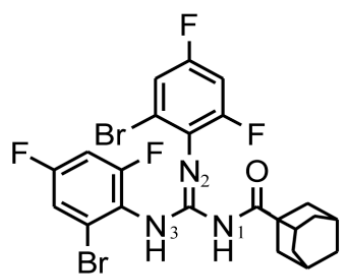

11, H-BDF (603.2)

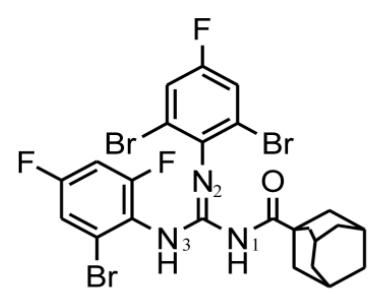

12 (664.2)<smiles>O=C(N/C(=N/c1ccccc1[N+](=O)[O-])Nc1ccccc1[N+](=O)[O-])c1ccccc1</smiles>

13 (463.5)

Figure 2. Chemical structure of guanidine derivative compounds 11-13. Molecular weights (g/mol) are shown in parentheses. 
Table 1. Activities of newly obtained compounds and common antibiotics used in clinical treatments against Pseudomonas aeruginosa PAO1 and Burkholderia cenocepacia J2315.

\begin{tabular}{|c|c|c|c|c|c|c|c|c|c|}
\hline \multirow{2}{*}{ Entry } & \multirow{2}{*}{$\mathrm{R}_{1}$} & \multirow{2}{*}{$\mathbf{R}_{2}$} & \multirow{2}{*}{$\mathbf{R}_{3}$} & \multirow{2}{*}{$\begin{array}{c}\begin{array}{c}\text { Molecular Weight } \\
(\mathrm{g} / \mathrm{mol})\end{array} \\
\end{array}$} & \multirow{2}{*}{$\begin{array}{l}\text { Chemical } \\
\text { Structure }\end{array}$} & \multicolumn{2}{|c|}{ P. aeruginosa PAO1 } & \multicolumn{2}{|c|}{ B. cenocepacia J2315 } \\
\hline & & & & & & MIC ( $\mu \mathrm{g} / \mathrm{mL})$ & MBC $(\mu \mathrm{g} / \mathrm{mL})$ & MIC ( $\mu \mathrm{g} / \mathrm{mL})$ & MBC $(\mu \mathrm{g} / \mathrm{mL})$ \\
\hline 1 & $\mathrm{C}_{10} \mathrm{H}_{15}{ }^{\mathrm{a}}$ & $\mathrm{C}_{6} \mathrm{H}_{11}$ & & 320.19 & & $>128$ & nd & $>128$ & nd \\
\hline 2 & $\mathrm{C}_{10} \mathrm{H}_{15}$ a & $\mathrm{C}_{6} \mathrm{H}_{5}$ & - & 314.45 & & $>128$ & nd & $>128$ & nd \\
\hline 3 & $\mathrm{C}_{10} \mathrm{H}_{15}{ }^{\mathrm{a}}$ & 3-F-4- $\mathrm{CH}_{3}-\mathrm{C}_{6} \mathrm{H}_{3}$ & - & 385.97 & & & nd & $>128$ & nd \\
\hline 4 & $\mathrm{C}_{10} \mathrm{H}_{15}{ }^{\mathrm{a}}$ & $2-\mathrm{NO}_{2}-\mathrm{C}_{6} \mathrm{H}_{4}$ & - & 359.44 & & $>128$ & nd & $>128$ & nd \\
\hline 5 & $\mathrm{C}_{10} \mathrm{H}_{15}{ }^{\mathrm{a}}$ & $4-\mathrm{CH}_{3} \mathrm{CO}-\mathrm{C}_{6} \mathrm{H}_{4}$ & - & 356.47 & & & nd & $>128$ & nd \\
\hline 6 & $\mathrm{C}_{10} \mathrm{H}_{15}{ }^{\mathrm{a}}$ & $2,3-\mathrm{di}-\mathrm{Cl}-\mathrm{C}_{6} \mathrm{H}_{3}$ & - & 383.34 & & & nd & $>128$ & nd \\
\hline 7 & $\mathrm{C}_{10} \mathrm{H}_{15}{ }^{\mathrm{a}}$ & 2-Br-4,6-di-F- ${ }_{6} \mathrm{H}_{2}$ & - & 428.32 & & $>128$ & nd & $>128$ & nd \\
\hline 8 & $\mathrm{C}_{10} \mathrm{H}_{7} \mathrm{~b}$ & $2-\mathrm{Br}-4,6$-di-F- ${ }_{6} \mathrm{H}_{2}$ & - & 421.97 & & & nd & $>128$ & nd \\
\hline 9 & 2,4-di-Cl- $\mathrm{C}_{6} \mathrm{H}_{3}$ & 2-Br-4,6-di-F- $\mathrm{C}_{6} \mathrm{H}_{2}$ & - & 439.88 & & $>128$ & nd & $>128$ & nd \\
\hline
\end{tabular}


Table 1. Cont.

\begin{tabular}{|c|c|c|c|c|c|c|c|c|c|}
\hline \multirow{2}{*}{ Entry } & \multirow{2}{*}{$\mathbf{R}_{1}$} & \multirow{2}{*}{$\mathbf{R}_{2}$} & \multirow{2}{*}{$\mathbf{R}_{3}$} & \multirow{2}{*}{$\begin{array}{c}\text { Molecular Weight } \\
(\mathrm{g} / \mathrm{mol})\end{array}$} & \multirow{2}{*}{$\begin{array}{l}\text { Chemical } \\
\text { Structure }\end{array}$} & \multicolumn{2}{|c|}{ P. aeruginosa PAO1 } & \multicolumn{2}{|c|}{ B. cenocepacia J2315 } \\
\hline & & & & & & MIC $(\mu \mathrm{g} / \mathrm{mL})$ & $\mathrm{MBC}(\mu \mathrm{g} / \mathrm{mL})$ & MIC $(\mu \mathrm{g} / \mathrm{mL})$ & MBC $(\mu \mathrm{g} / \mathrm{mL})$ \\
\hline 10 & $4-\mathrm{CH}_{3}-\mathrm{C}_{6} \mathrm{H}_{4}$ & 2-Br-4,6-di-F- $\mathrm{C}_{6} \mathrm{H}_{2}$ & - & 385.97 & & $>128$ & nd & $>128$ & nd \\
\hline 11 & $\mathrm{C}_{10} \mathrm{H}_{15}{ }^{\mathrm{a}}$ & 2-Br-4,6-di-F- $\mathrm{C}_{6} \mathrm{H}_{2}$ & 2-Br-4,6-di-F- $\mathrm{C}_{6} \mathrm{H}_{2}$ & 603.2 & & 0.5 & 4 & 2 & 8 \\
\hline Tobramycin & & & & 467.51 & & 2 & 2 & $>128$ & $>128$ \\
\hline Meropenem & & & & 383.46 & & 1 & 4 & 8 & 64 \\
\hline Ceftazimide & & & & 546.57 & & 2 & 2 & 16 & 128 \\
\hline
\end{tabular}

a 1-adamantyl, ${ }^{\text {b-naphthyl }}$ 


\subsection{Bacterial Strains}

The antibacterial activity of the compounds was tested against the reference strains Escherichia coli ATCC25922, Bordetella bronchiseptica 9.73H+ [27], Pseudomonas aeruginosa ATCC15692, Burkholderia cenocepacia J2315, Pandorea apista DSM16535, Staphyloccocus aureus ATCC6538, Bacillus cereus ATCC10876. A total of forty non-fermenting Gram-negative bacilli and two Methicillin-Resistant Staphylococcus aureus (MRSA) clinical isolates collected from sputum samples of patients with CF attended at different hospitals and CF Centers in the period 2004 to 2017 were used in this study. They were selected from the collection of microorganisms CAMPA (Colección Argentina de Microorganismos Patógenos y Ambientales) of CINDEFI, at the Faculty of Exact Sciences in La Plata University [28]. All Bcc isolates were identified by PCR-recA technology (amplification, PCR-recA RFLP HaeIII, and sequencing). Additionally his $A, g y r B$, or other gene from the current multilocus sequence typing (MLST) scheme were sequenced when the identification remained ambiguous [29,30]. The isolates were maintained both as lyophilized and frozen at $-80{ }^{\circ} \mathrm{C}$ in Trypticase-soy broth with $10 \%(v / v)$ glycerol until further analysis.

\subsection{Antimicrobial Activity Assays}

The in vitro susceptibility tests (Minimal inhibitory concentration (MIC) and minimal bactericidal concentration (MBC) tests) were determined using the micro-dilution method according to guidelines of the Clinical and Laboratory Standards Institute (CLSI) [31]. Briefly, serial two-fold dilutions of each compound were prepared (final volume of $50 \mu \mathrm{L}$ ) in 96-well polypropylene microtiter plates (Sarstedt, Nümbrecht, Germany) with Mueller Hinton (MH) broth. Each dilution series included control wells without any compound and control wells without bacteria. Then, a total of $50 \mu \mathrm{L}$ of the adjusted inoculum (approximately $5 \times 10^{5}$ cells $/ \mathrm{mL}$ ) in $\mathrm{MH}$ broth was added to each well. The MIC was taken as the lowest concentration of antimicrobial compound resulting in the complete inhibition of visible growth after $18 \mathrm{~h}$ of incubation at $37^{\circ} \mathrm{C}$. Minimal bactericidal concentration (MBC) assay was performed following MIC assay. After reporting the MIC assay value, $10 \mu \mathrm{L}$ aliquots of the medium were taken from wells with no visible bacterial growth. These were plated on LB agar and incubated for $24 \mathrm{~h}$ to allow colony growth. The lowest concentration of the compound at which no growth occurred on LB plates was denoted as the MBC. Results are mean values of at least two independent determinations.

\subsection{Checkerboard Assay}

The activity of compound $\mathbf{1 1}$ in combination with meropenem, tobramycin and ciprofloxcin was analyzed using the checkerboard broth dilution method [32] to determine the fractional inhibitory concentration indices (FICIs), calculated as: FICI $=\left(\mathrm{MIC}_{\mathbf{H}-\mathrm{BDF}}{ }^{\mathrm{comb}} / \mathrm{MIC}_{\mathbf{H}-\mathrm{BDF}}{ }^{\text {alone }}\right)+$ $\left(\mathrm{MIC}_{\text {antibiotic }}{ }^{\mathrm{comb}} / \mathrm{MIC}_{\text {antibiotic }}{ }^{\text {alone }}\right.$ ) (comb, combination). The calculated FICI was interpreted as synergistic $(\mathrm{FICI} \leq 0.5)$, additive $(0.5<\mathrm{FICI}<1)$, indifferent $(1 \leq \mathrm{FICI}<4.0)$, or antagonistic $(\mathrm{FICI} \geq 4.0)$, according to the previously published methods [33].

\subsection{Cytotoxicity Assays}

A trypan blue exclusion assay [34] was performed to check the cytotoxicity of compound 11 against THP-1 human monocytic leukemia cells (ATCC, TIB-202, Manassas, VA, USA) and A549 alveolar epithelial cells (ATCC, CCL185, Rockville, MD, USA). Cells were routinely maintained in Complete Medium RPMI-1640 and Dulbecco's Modified Eagle's medium (DMEM), respectively, supplemented with $10 \%$ heat-inactivated fetal bovine serum (FBS). For the cytotoxicity assay, cells were seeded at a density of $5 \times 10^{4}$ per well in a 96 well plate and were incubated with serial dilutions of compound 11 to a total of $200 \mu \mathrm{L}$, at $37{ }^{\circ} \mathrm{C}$ in a humidified atmosphere of $5 \% \mathrm{CO}_{2}$ for $24 \mathrm{~h}$. Two negative controls were included: cells in drug-free culture media and cells treated for $24 \mathrm{~h}$ with the maximum concentration of the drug solvent used in the experiment ( $\%$ dimethyl sulfoxide). 
Cells were subsequently stained with $0.2 \%$ trypan blue and incubated for $3 \mathrm{~min}$ at room temperature. The number of dye-excluding cells was counted by microscopy. A minimum of 200 cells were counted and the percent viability was calculated in comparison to the control. The IC50 value was defined as the highest drug concentration at which $50 \%$ of the cells are viable relative to the control. Results are mean values of at least five independent determinations. The selectivity index (SI) was calculated as the ratio of IC50 and the MIC [35].

\section{Results and Discussion}

\subsection{Chemistry}

A series of 11 novel closely related compounds belonging to the thiourea family (compounds 1-10) and a guanidine derivative (11) was prepared (Figure 1 and Table 1). Primary amines substituted with different electron withdrawing groups were subjected to the addition reaction with isothiocyanates in order to be transformed into the corresponding thioureas by using the general method originally proposed by Douglas and Dains [22] (Figure 1). The substitution on both nitrogen positions (1 and 3 ) of the thiourea group was varied in order to better understand the role of different substituents in the biological activity. To rationalize this aspect, a series of closely related 1-(adamantane-1-carbonyl)-3-mono substituted thioureas was firstly prepared by taking into account the well-known capacity of the adamantyl group to enhance antibacterial activity [36-40]. Thus, several thioureas were prepared bearing the adamantyl group in $\mathrm{R}_{1}$ (compounds 1-7, Table 1).

Moreover, taking into account the improvement in antibacterial activity exerted by the presence of phenyl groups substituted with electron withdrawing groups [16,19-21], a second group of thioureas (compounds 7-10, Table 1) was substituted in $\mathrm{R}_{2}$ with the 2-bromo-4,6-difluoro-phenyl group. Finally, the effect of replacing the thiocarbonyl $(C=S)$ with aryl-guanidino functionality $(\mathrm{Ar}-\mathrm{N}=\mathrm{C})$ was evaluated in compound 7 , in which $\mathrm{N}-3$ of the guanidine was substituted with the 2-bromo-4,6-difluoro-phenyl group. To this end, the procedure proposed by Vencato et al. [26] was applied and the acyl thiourea derivatives were treated with mercury(II) chloride under basic conditions in the presence of 2-bromo-4,6-difluoroaniline to produce the corresponding guanidine derivative (compound 11, Figure 1 and Table 1) [26,41].

Obtained compounds were purified by flash chromatography. FTIR, ${ }^{1} \mathrm{H}-\mathrm{NMR}$ and ${ }^{13} \mathrm{C}-\mathrm{NMR}$ spectra and elemental analysis confirmed the identity of the products (see Materials and Methods). In the ${ }^{1} \mathrm{H}-\mathrm{NMR}$ of most of the compounds, the characteristic signals of adamantyl moiety: a $6 \mathrm{H}$ quartet at $\delta=1.75-1.79$ ppm (adamantane- $\mathrm{CH}_{2}$ ), a $6 \mathrm{H}$, singlet at 1.95-1.98 (adamantane- $\left.\mathrm{CH}_{2}\right)$ and a $3 \mathrm{H}$, singlet around 2.08 ppm (adamantane- $\mathrm{CH}$ ), besides $\mathrm{N}-\mathrm{H}$ amide and thioamide singlets at $\delta=8.5-8.7$ and 12.7-13.0 ppm were clearly observed. In the ${ }^{13} \mathrm{C}-\mathrm{NMR}$, characteristic signals for adamantyl moiety at $\delta=27.7,36.1-36.4,38.6-38.5$ and $41.5 \mathrm{ppm}$, as well those at $\delta=170-179$ for carbonyl and $\delta=178-182$ ppm for thiocarbonyl carbons, were observed. The acyl thioureas were also characterized by their IR spectra, with intense absorptions around 3300-3400 (vNH), $1670(v \mathrm{C}=\mathrm{O}), 1580(\delta \mathrm{NH})$, and $1380(v C=S) \mathrm{cm}^{-1}[24,42,43]$.

The guanidine derivative $\mathbf{1 1}$ was characterized by two typical NH absorptions at ca. 3400 and $3240 \mathrm{~cm}^{-1}$, the $\mathrm{C}=\mathrm{O}$ stretching at around $1670 \mathrm{~cm}^{-1}$ and the absence of thiocarbonyl stretching when the FTIR spectra are compared with the corresponding thiourea reagent. The characteristic $\mathrm{C}=\mathrm{N}$ stretching modes of the guanidine group are observed as an intense absorption at ca. $1575 \mathrm{~cm}^{-1}$. In ${ }^{1} \mathrm{H}-\mathrm{NMR}$, two broad NH singlets appeared besides the aromatic protons. The carbonyl carbons are observed at $178-179 \mathrm{ppm}$ in the ${ }^{13} \mathrm{C}$-NMR spectrum, while the $(\mathrm{C}=\mathrm{N}-\mathrm{Ar})$ appeared upfield at $174 \mathrm{ppm}$ compared to the thiocarbonyl carbon. 


\subsection{Biological Activity}

\subsubsection{Antimicrobial Evaluation of Newly Synthesized Compounds}

All obtained compounds were tested in vitro for their MIC and MBC against two reference Gram-negative non-fermentative bacilli strains, Pseudomonas aeruginosa PAO1 and Burkholderia cenocepacia J2315. These species play a critical role in morbidity and mortality associated with CF and they were selected on the basis of their high level of resistance to a variety of antimicrobial substances [44-47]. The results of antimicrobial activity are summarized in Table 1 . The MIC and MBC values of meropenem, tobramycin and ceftazidime, three commonly used antibiotics for the treatment of chronic pulmonary bacterial infections [48], were analyzed in parallel. It is apparent from the results that only the guanidine derivative 11, namely H-BDF, showed a MIC value less than $2 \mu \mathrm{g} / \mathrm{mL}$, and comparable or superior activity than standard drugs. Interestingly, this compound has the lowest MIC and MBC against B. cenocepacia J2315, a strain particularly resistant to meropenem [44].

A first look into structural activity relationship (SAR) indicates that, independent of the halogens introduced in the phenyl group, thiourea derivatives have poor or no antimicrobial activity. However, the replacement of thiourea in compound 7 for the guanidine group (compound 11) greatly improves antimicrobial activity. We next evaluated the impact of introducing changes in the phenyl ring of compound 11 in the biological activity. To this end, the guanidine derivatives 12 and 13 (Figure 2) were synthesized and characterized. Compound 13 was designed to evaluate the effect of changing the substitution of the halophenyl groups by the incorporation of another electron withdrawing group (nitro) in N-2 and N-3, whereas compound 12 evaluates the effect of introducing a small change in N-3 by the substitution of bromine by fluorine in position 6 .

The antimicrobial activity of the new compounds was tested against P. aeruginosa PAO1 and $B$. cenocepacia J2315 as well as other Gram-negative and Gram-positive reference strains. As shown in Table 2, when the phenyl group substituent of compound $\mathbf{1 1}$ was altered by the introduction of a nitro group at the meta position (compound 13), the guanidine derivate completely lost its inhibition potency, suggesting that not only the guanidine group but also the identity and/or position of the phenyl substitutions are decisive for the antibacterial activity. Moreover, whereas compound $\mathbf{1 1}$ exhibited very good inhibitory and bactericidal activity against all tested strains, compound 12, in which the 2-bromo-4,6-difluoro-phenyl group in N-2 was substituted by 2,6-dibromo-4-fluoro-phenyl ring, showed only moderate microbicidal activity, suggesting that the presence of fluorine atom in position 6 of the phenyl group in N-2 is critical to ensure high inhibition and bactericidal potency.

Table 2. Anitmicrobial activities of new compounds 11, 12 and 13 against Gram-negative and Gram-positive bacteria-minimal inhibitory concentrations (MIC, $\mu \mathrm{g} / \mathrm{mL}$ ) and minimal bactericidal concentration $(\mathrm{MBC}, \mu \mathrm{g} / \mathrm{mL})$.

\begin{tabular}{ccccccc}
\hline Compound & $\mathbf{1 1}$ (H-BDF) & $\mathbf{1 2}$ & $\mathbf{1 3}$ & Tobramycin & Meropenem & Ceftazimide \\
\hline Organism & MIC/MBC & MIC/MBC & MIC/MBC & MIC/MBC & MIC/MBC & MIC/MBC \\
\hline \multicolumn{7}{c}{ Gram-negative bacteria } \\
\hline Bordetella bronchiseptica $9.73 H+$ & $0.5 / 2$ & $16 / 64$ & $>128 />128$ & $64 / 64$ & $0.125 / 0.25$ & $8 / 64$ \\
Escherichia coli ATCC25922 & $1 / 2$ & $64 / 64$ & $>128 />128$ & $16 / 16$ & $0.03125 / 0.0625$ & $1 / 1$ \\
Pseudomonas aeruginosa PAO1 & $0.5 / 4$ & $32 />128$ & $>128 />128$ & $2 / 2$ & $1 / 4$ & $2 / 2$ \\
Burkholderia cenocepacia J2315 & $2 / 8$ & $64 / 128$ & $>128 />128$ & $>128 />128$ & $8 / 64$ & $16 / 128$ \\
Pandorea apista DSM16535 & $1 / 2$ & $64 / 128$ & $>128 />128$ & $32 / 128$ & $>128 /$ nd & $128 /$ nd \\
\hline & \multicolumn{7}{c}{ Gram-positive bacteria } \\
\hline Staphyloccocus aureus ATCC6538 & $0.25 / 1$ & $8 / 64$ & $>128 />128$ & $2 / 2$ & $<0.125 /<0.25$ \\
Bacillus cereus ATCC10876 & $2 / 2$ & $64 / 64$ & $>128 />128$ & $8 / 32$ & $</ 8$ \\
\hline
\end{tabular}

nd: no data 


\subsubsection{Cytotoxic Evaluation of $\mathbf{H}-\mathbf{B D F}$}

As limited human cellular toxicity is an important feature for an antibiotic compound, the toxicity of H-BDF was evaluated using the human monocytic leukemia cell line THP-1 and the human lung epithelial cell line A549, commonly employed in toxicity evaluation of new compounds for pulmonary application [49,50]. The IC50 for compound 11 was $38.4 \pm 5.4 \mu \mathrm{g} / \mathrm{mL}$ for A549 and $15.5 \pm 3.1 \mu \mathrm{g} / \mathrm{mL}$ for THP-1 cells. On the basis of the MIC and IC50 values, the selectivity indices were calculated for standard strains (Table 3). It is generally considered that the ratio for a good therapeutic index for a drug should be $>10$, which is a cut-off point ensuring that overdose does not put the life of the patient in danger [35]. Good SI values were obtained with compound 11 suggesting that H-BDF can be considered as a promising antibacterial agent.

Table 3. Selective Indices (SI) of compound 11 against different cell lines.

\begin{tabular}{ccc}
\hline & & Cells \\
\hline Organisms & A549 & THP-1 \\
\hline Gram-negative bacteria & & \\
\hline Bordetella bronchiseptica 9.73H+ & 76.8 & 30.9 \\
Escherichia coli ATCC25922 & 38.4 & 15.45 \\
Pseudomonas aeruginosa PAO1 & 76.8 & 30.9 \\
Burkholderia cenocepacia J2315 & 19.2 & 7.7 \\
Pandorea apista DSM16535 & 38.4 & 15.45 \\
\hline Gram-positive bacteria & & \\
\hline Staphyloccocus aureus ATCC6538 & 153.6 & 61.8 \\
Bacillus cereus ATCC10876 & 19.2 & 7.7 \\
\hline
\end{tabular}

\subsubsection{Synergistic Effects between $\mathbf{H}-\mathbf{B D F}$ and Conventional Antibiotics}

Developments of alternate antibacterial strategies to potentiate the antimicrobial activity of conventional antibiotics have become increasingly important due to the emerging threat of multi-drug resistant infection [51]. As many clinical isolates exhibit resistance to meropenem, ciprofloxacin and tobramycin, three of the different classes of antibiotics commonly used to treat CF pulmonary exacerbations [52], we next studied the ability of H-BDF to potentiate the antimicrobial activity of these antibiotics toward the multidrug-resistant strain B. cenocepacia J2315. To this end, the relationship between H-BDF and meropenem, tobramycin, and ciprofloxacin was assessed via a standard checkerboard assay [29]. Treatment with H-BDF reduced the minimum inhibitory concentration of ciprofloxacin and meropenem below their clinical sensitivity breakpoints $(\leq 4 \mu \mathrm{g} / \mathrm{mL}$ and $\leq 1 \mu \mathrm{g} / \mathrm{mL}$, respectively). Fractional inhibitory concentration calculations revealed that $\mathbf{H}-\mathbf{B D F}$ exhibited a synergistic interaction with meropenem and ciprofloxacin with FICIs values of 0.3 and 0.4 , respectively, and an additive interaction with tobramycin with a FICI value of 0.75 . This preliminary study suggests that in addition to being used as antimicrobial agent alone, H-BDF has the potential to be used in combination with other antibiotics.

3.2.4. Activity of Compound H-BDF against Multidrug-Resistant Clinical Isolates Recovered from Respiratory Samples of CF Patients

Respiratory infections with opportunistic pathogens with intrinsic antibiotic resistance to most clinically available antimicrobials are life-threatening in patients with CF [53-55]. Although P. aeruginosa and $S$. aureus remain the most common pathogens in CF lung infections, other bacteria such as species within the Bcc, Stenotrophomonas maltophilia, and Achromobacter xylosoxidans, have emerged as significant opportunistic human pathogens in the last decades [56-59]. To investigate whether the guanidine derivative $\mathbf{H - B D F}$ would have clinical utility against current multidrug resistant bacteria, we determined the MIC and MBC of compound $\mathbf{1 1}$ against thirty eight Bcc clinical isolates, 
one Achromobacter xylosoxidans, one Stenotrophomonas maltophilia and two MRSA recovered from sputum samples of CF patients and selected on the basis of their high level of resistance to a variety of antimicrobial substances [55] (Table 4). MIC values of compound H-BDF were generally lower than those of meropenem, ceftazimide and tobramycin. In total, $69 \%$ of Bcc clinical isolates had H-BDF MIC values less than or equal to $4 \mu \mathrm{g} / \mathrm{mL}$ whereas only $41 \%$ of isolates were classified as susceptible to meropenem (MIC values $\leq 4 \mu \mathrm{g} / \mathrm{mL}$ ), $49 \%$ were classified as susceptible to ceftazimide (MIC values $\leq 8 \mu \mathrm{g} / \mathrm{mL}$ ), and $2.6 \%$ of isolates were classified as susceptible to tobramycin (MIC values $\leq 4 \mu \mathrm{g} / \mathrm{mL}$ ). The activity of compound H-BDF against B. cenocepacia strains was impressive, with $92 \%$ susceptible at $4 \mu \mathrm{g} / \mathrm{mL}$ compared with only $31 \%$ susceptible to meropenem at $4 \mu \mathrm{g} / \mathrm{mL}$, and $69 \%$ susceptible to ceftazimide at $8 \mu \mathrm{g} / \mathrm{mL}$ (Table 4 ). Interestingly, some clinical isolates were resistant to more than 16 antibiotics, such as B. seminalis CBC040 [55] had H-BDF MIC values $\leq 4 \mu \mathrm{g} / \mathrm{mL}$. Indeed, H-BDF was active against two methicillin-resistant $S$. aureus clinical isolates with MIC values varying from 1 to $2 \mu \mathrm{g} / \mathrm{mL}$. In conclusion, compound H-BDF was active in vitro against a significant number of multi-resistant clinical isolates recovered from CF patients. 
Table 4. Microbial susceptibility of multi-resistant isolates recovered from patients with cystic fibrosis.

\begin{tabular}{|c|c|c|c|c|c|c|c|c|}
\hline \multirow[b]{2}{*}{ Clinical Isolates $^{a}$} & \multicolumn{2}{|c|}{ H-BDF } & \multicolumn{2}{|c|}{ Tobramycin } & \multicolumn{2}{|c|}{ Meropenem } & \multicolumn{2}{|c|}{ Ceftazidime } \\
\hline & MIC $(\mu \mathrm{g} / \mathrm{mL})$ & $\mathrm{MBC}(\mu \mathrm{g} / \mathrm{mL})$ & MIC $(\mu \mathrm{g} / \mathrm{mL})$ & $\mathrm{MBC}(\mu \mathrm{g} / \mathrm{mL})$ & MIC $(\mu \mathrm{g} / \mathrm{mL})$ & MBC $(\mu \mathrm{g} / \mathrm{mL})$ & MIC ( $(\mu \mathrm{g} / \mathrm{mL})$ & $\mathrm{MBC}(\mu \mathrm{g} / \mathrm{mL})$ \\
\hline \multicolumn{9}{|l|}{ Achromobacter xylosoxidans } \\
\hline $\begin{array}{l}\text { A. xylosoxidans HNA } 001 \\
\text { Burkholderia cenocepacia }\end{array}$ & 0.125 & 0.25 & $\mathrm{R}$ & nd & S & 8 & S & nd \\
\hline B. сепосерасіа CAMPA 669 & 0.25 & 2 & $\mathrm{~s}$ & nd & $\mathrm{s}$ & nd & $\mathrm{R}$ & nd \\
\hline B. cenocepacia CAMPA 1533 & 4 & 16 & $\mathrm{R}$ & nd & $\mathrm{R}$ & 64 & $\mathrm{~s}$ & 16 \\
\hline B. cenocepacia CAMPA 1194 & 2 & 4 & $\mathrm{R}$ & nd & $\mathrm{R}$ & nd & $\mathrm{R}$ & nd \\
\hline B. сепосерасіа CAMPA 544 & 2 & 8 & $\mathrm{R}$ & nd & $\mathrm{R}$ & nd & $\mathrm{s}$ & 8 \\
\hline B. сепосерасіа CAMPA 1771 & 8 & 16 & $\mathrm{R}$ & nd & I & 32 & $\mathrm{R}$ & nd \\
\hline B. сепосерасіа CAMPA 817 & 2 & 8 & $\mathrm{R}$ & nd & $\mathrm{R}$ & nd & $\mathrm{s}$ & 8 \\
\hline B. cenocepacia CAMPA 548 & 2 & 4 & $\mathrm{R}$ & nd & $\mathrm{R}$ & nd & $\mathrm{s}$ & 8 \\
\hline B. cenocepacia CAMPA 825 (CВC 033) $^{\text {b }}$ & 4 & 16 & $\mathrm{R}$ & nd & I & nd & $\mathrm{s}$ & 32 \\
\hline B. cenocepacia CAMPA538 (СВC 035) ${ }^{\mathrm{b}}$ & 2 & 4 & $\mathrm{R}$ & nd & I & 16 & $\mathrm{~s}$ & 16 \\
\hline B. сепосерасіа CAMPA 817 & 2 & 8 & $\mathrm{R}$ & nd & $\mathrm{R}$ & nd & $\mathrm{s}$ & 16 \\
\hline B.cenocepacia CAMPA 531 & 1 & 4 & $\mathrm{R}$ & nd & $\mathrm{S}$ & nd & $\mathrm{s}$ & nd \\
\hline B.cenocepacia CAMPA 993 (СВC 024) & 1 & 4 & $\mathrm{R}$ & nd & S & nd & S & nd \\
\hline B.cenocepacia HE001 & 4 & 64 & $\mathrm{R}$ & nd & $\mathrm{R}$ & nd & $\mathrm{R}$ & nd \\
\hline \multicolumn{9}{|l|}{ Burkholderia cepacia } \\
\hline B. серасіа CAMPA 545 & 4 & 16 & $\mathrm{R}$ & nd & $\mathrm{R}$ & nd & $\mathrm{S}$ & 16 \\
\hline B. cepacia CAMPA 233 (СBC 012) b & 2 & 4 & $\mathrm{R}$ & nd & $\mathrm{s}$ & 8 & $\mathrm{~s}$ & 16 \\
\hline B. cepacia CAMPA 260 & 32 & nd & $\mathrm{R}$ & nd & $\mathrm{R}$ & 32 & $\mathrm{R}$ & nd \\
\hline B. серасіа CAMPA 914 & 32 & nd & $\mathrm{R}$ & nd & $\mathrm{R}$ & 32 & $\mathrm{R}$ & 64 \\
\hline B. cepacia CAMPA 886 & 32 & nd & $\mathrm{R}$ & nd & $\mathrm{R}$ & 32 & $\mathrm{R}$ & 128 \\
\hline B. серасіа CAMPA 998 & 32 & nd & $\mathrm{R}$ & nd & $\mathrm{R}$ & 64 & $\mathrm{~s}$ & 32 \\
\hline B. серасіа CAMPA 1039 & 64 & nd & $\mathrm{R}$ & nd & $\mathrm{R}$ & 32 & $\mathrm{R}$ & nd \\
\hline B. cepacia CAMPA 853 (СBC 001) b & 32 & nd & $\mathrm{R}$ & nd & I & 64 & I & 64 \\
\hline B. cepacia CAMPA 860 (CBC 007) b & 64 & nd & $\mathrm{R}$ & nd & I & 64 & $\mathrm{R}$ & 64 \\
\hline B. cepacia CAMPA 660 & 4 & 8 & $\mathrm{R}$ & nd & $\mathrm{s}$ & 4 & $\mathrm{R}$ & nd \\
\hline B. cepacia CAMPA $721\left(\right.$ СВC 011) ${ }^{b}$ & 2 & 32 & $\mathrm{R}$ & nd & S & 64 & $\mathrm{R}$ & nd \\
\hline \multicolumn{9}{|l|}{ Burkholderia contaminans } \\
\hline B. contaminans HNBC001 & 0.25 & 1 & $\mathrm{R}$ & nd & $\mathrm{R}$ & nd & $\mathrm{S}$ & nd \\
\hline \multicolumn{9}{|l|}{ Burkholderia multivorans } \\
\hline B. multivorans CAMPA $661(\mathrm{CBC} 015)^{\mathrm{b}}$ & 2 & 4 & $\mathrm{R}$ & nd & $\mathrm{s}$ & 4 & S & 8 \\
\hline B. multivorans CAMPA 1530 & 2 & 8 & $\mathrm{R}$ & nd & $\mathrm{R}$ & nd & $\mathrm{s}$ & 4 \\
\hline B. multivorans CAMPA 647 (CBC 017) b & 4 & 4 & $\mathrm{R}$ & nd & $\mathrm{s}$ & 4 & $\mathrm{~s}$ & 8 \\
\hline B. multivorans CAMPA $653(\mathrm{CBC} 018)^{\mathrm{b}}$ & 2 & 8 & $\mathrm{R}$ & nd & $\mathrm{s}$ & 4 & $\mathrm{~s}$ & 8 \\
\hline B. multivorans CAMPA 623(CBC 019) ${ }^{\mathrm{b}}$ & 2 & 8 & $\mathrm{R}$ & nd & $\mathrm{s}$ & 8 & $\mathrm{R}$ & nd \\
\hline B. multivorans CAMPA 832 (CBC 020) b & 4 & 16 & $\mathrm{R}$ & nd & $\mathrm{s}$ & 32 & $\mathrm{R}$ & nd \\
\hline B. multivorans CAMPA 987 (CBC 021) b & 2 & 4 & $\mathrm{R}$ & nd & $\mathrm{s}$ & 8 & $\mathrm{R}$ & nd \\
\hline B. multivorans CAMPA 997 (CBC 022) b & 4 & 8 & $\mathrm{R}$ & nd & $\mathrm{s}$ & 8 & $\mathrm{R}$ & nd \\
\hline
\end{tabular}


Table 4. Cont.

\begin{tabular}{|c|c|c|c|c|c|c|c|c|}
\hline \multirow[b]{2}{*}{ Clinical Isolates ${ }^{a}$} & \multicolumn{2}{|c|}{ H-BDF } & \multicolumn{2}{|c|}{ Tobramycin } & \multicolumn{2}{|c|}{ Meropenem } & \multicolumn{2}{|c|}{ Ceftazidime } \\
\hline & MIC $(\mu \mathrm{g} / \mathrm{mL})$ & MBC $(\mu \mathrm{g} / \mathrm{mL})$ & $\operatorname{MIC}(\mu \mathrm{g} / \mathrm{mL})$ & MBC $(\mu \mathrm{g} / \mathrm{mL})$ & MIC $(\mu \mathrm{g} / \mathrm{mL})$ & $\operatorname{MBC}(\mu \mathrm{g} / \mathrm{mL})$ & MIC ( $\mu \mathrm{g} / \mathrm{mL})$ & $\mathrm{MBC}(\mu \mathrm{g} / \mathrm{mL})$ \\
\hline \multicolumn{9}{|l|}{ Burkholderia seminalis } \\
\hline B. seminalis CAMPA 231 & 32 & nd & $\mathrm{R}$ & nd & I & nd & $\mathrm{R}$ & 32 \\
\hline B. seminalis CAMPA 261 (CBC 039) b & 32 & nd & $\mathrm{R}$ & nd & $\mathrm{s}$ & 16 & $\mathrm{~s}$ & 16 \\
\hline B. seminalis CAMPA 475 (CBC 040) b & 4 & 8 & $\mathrm{R}$ & nd & I & nd & $\mathrm{R}$ & nd \\
\hline B. seminalis CAMPA 227 & 1 & 8 & $\mathrm{R}$ & nd & $\mathrm{R}$ & nd & $\mathrm{R}$ & nd \\
\hline \multicolumn{9}{|l|}{ Burkholderia vietnamiensis } \\
\hline B. vietnamiensis CAMPA $992\left(\right.$ CBC 038) ${ }^{b}$ & 32 & nd & $\mathrm{R}$ & nd & $\mathrm{S}$ & 8 & $\mathrm{~S}$ & 16 \\
\hline \multicolumn{9}{|l|}{ Staphylococcus aureus } \\
\hline S. aureus CAMPA 1909 & 2 & 16 & 128 & nd & $>128$ & nd & $>128$ & nd \\
\hline S. aureus CAMPA 1908 & 1 & 4 & 32 & $>128$ & $>128$ & nd & $>128$ & nd \\
\hline \multicolumn{9}{|l|}{ Stenotrophomonas maltophilia } \\
\hline S. maltophilia CAMPA 1911 & 2 & 16 & $>128$ & nd & $>128$ & nd & $>128$ & nd \\
\hline
\end{tabular}

nd = non-determined. $\mathrm{R}=$ resistant, $\mathrm{I}=$ intermediate, $\mathrm{S}=$ sensible (according to the criteria set up by the CLSI). Meropenem $(<4 \mu \mathrm{g} / \mathrm{mL} \mathrm{S,} 8 \mu \mathrm{g} / \mathrm{mL} \mathrm{I}, \geq 16 \mu \mathrm{g} / \mathrm{mL} \mathrm{R})$. Ceftazidime $\left(\leq 8 \mu \mathrm{g} / \mathrm{mL} \mathrm{S}, 16 \mu \mathrm{g} / \mathrm{mL} \mathrm{I}, \geq 32 \mu \mathrm{g} / \mathrm{mL}\right.$ R). Tobramycin $\left(\leq 4 \mu \mathrm{g} / \mathrm{mL} \mathrm{S}, 8 \mu \mathrm{g} / \mathrm{mL} \mathrm{I}, \geq 16 \mu \mathrm{g} / \mathrm{mL}\right.$ R). ${ }^{\text {a }}$ Isolates recovered from patients with chronic infections in the period $2004-2017$. ${ }^{\mathrm{b}}$ Isolates recovered from patients with cystic fibrosis whose complete antibiotic susceptibilities to 17 antimicrobial agents were previously reported (reference [55]). 


\section{Conclusions}

We have reported the synthesis and preliminary evaluation of the antimicrobial activity of 13 novel thiourea and guanidine derivatives. The results evidenced that H-BDF, a guanidine derivative bearing adamantane-1-carbonyl and two 2-bromo-4,6-di-fluoro-phenyl groups, can be considered as a promising antimicrobial agent, since it exhibited higher in vitro antibacterial potency against Gram-positive and Gram-negative reference strains than previously reported guanidine compounds $[10,11,15]$. Moreover, the novel compound was active in vitro against a panel of multidrug-resistant clinical isolates recovered from sputum samples of patients with CF. Preliminary studies further suggest that H-BDF was able to significantly potentiate antibacterial synergy with meropenem and ciprofloxacin. From the structure activity relationship, it can be concluded that the antimicrobial activity depends mainly on the presence of a guanidine group. It has been proposed that most of the biological properties of guanidine derivatives are related to their strong basicity due to efficient resonance stabilization of the charged protonated state. The pKa of H-BDF was not determined; however, it is expected that under physiological conditions, the guanidine group exists mainly in its protonated form [60]. We can hypothesize that under this state, the guanidine moiety may alter bacterial outer membrane permeability by binding to a negatively charged site in the lipopolysaccharide layer, causing cell death. This mechanism of action have been proposed for several guanidine derivatives with antibacterial activity [61]. Alternatively, the protonated forms may interact with the active site of proteins and enzymes altering its function [11]. By analyzing the role that different substituents exert in the antimicrobial activity, the importance of the halo-phenyl group in the guanidine moiety was also demonstrated. The substituted fluorine in position 6 of the phenyl group in N-2 may contribute to increase binding affinity and/or lipid solubility [18]. Also, the electron-withdrawing group may activate the guanidine binding moiety to enhance its interaction with amine groups present in the cell membrane. Future studies will be directed towards elucidating the targets of $\mathbf{H}-\mathbf{B D F}$ and the mechanisms of action.

Importantly, this compound displays low levels of cytotoxicity against THP-1 and A549 cell lines. Future research will be performed to evaluate its efficacy and safety in animal models of infection in order to validate its development as a novel antimicrobial.

\section{Patents}

“Antimicrobials compounds". Patent application PCT/IB2017/054870, August 2017.

Author Contributions: Aamer Saeed, Alejandra Bosch, Yanina Lamberti and Mauricio Federico Erben conceived and designed the experiments; Aamer Saeed, Diana L. Nossa González, Marisa Bettiol , and Yanina Lamberti performed the experiments; Aamer Saeed, Alejandra Bosch, Diana L. Nossa González, Yanina Lamberti and Mauricio Federico Erben analyzed the data; Alejandra Bosch and Marisa Bettiol contributed reagents and clinical isolates; Yanina Lamberti, Aamer Saeed, Alejandra Bosch, and Mauricio Federico Erben wrote the paper.

Acknowledgments: This work was supported by the Agencia Nacional de Promoción Científica y Tecnológica, ANPCyT (PICT-2013-2130) and Universidad Nacional de La Plata (11/X794), Argentina. Y.L. and M.F.E. are members of the Scientific Career of CONICET. D.L.N.G. is a doctoral fellow of CONICET. A.B. is member of CIC PBA.

Conflicts of Interest: The authors declare no conflict of interest.

\section{References}

1. Livermore, D.M. Has the era of untreatable infections arrived? J. Antimicrob. Chemother. 2009, 64, i29-i36. [CrossRef] [PubMed]

2. Boucher, H.W.; Talbot, G.H.; Bradley, J.S.; Edwards, J.E.; Gilbert, D.; Rice, L.B.; Scheld, M.; Spellberg, B.; Bartlett, J. Bad bugs, no drugs: No ESKAPE! An update from the Infectious Diseases Society of America. Clin. Infect. Dis. 2009, 48, 1-12. [CrossRef] [PubMed]

3. Walsh, T.R. Emerging carbapenemases: A global perspective. Int. J. Antimicrob. Agents 2010, 36, S8-S14. [CrossRef] 
4. McGowan, J.E., Jr. Resistance in nonfermenting gram-negative bacteria: Multidrug resistance to the maximum. Am. J. Med. 2006, 119, S29-S36. [CrossRef] [PubMed]

5. Speert, D.P.; Henry, D.; Vandamme, P.; Corey, M.; Mahenthiralingam, E. Epidemiology of Burkholderia cepacia complex in patients with cystic fibrosis, Canada. Emerg. Infect. Dis. 2002, 8, 181-187. [CrossRef] [PubMed]

6. Zhao, J.; Schloss, P.D.; Kalikin, L.M.; Carmody, L.A.; Foster, B.K.; Petrosino, J.F.; Cavalcoli, J.D.; VanDevanter, D.R.; Murray, S.; Li, J.Z.; et al. Decade-long bacterial community dynamics in cystic fibrosis airways. Proc. Natl. Acad. Sci. USA 2012, 109, 5809-5814. [CrossRef] [PubMed]

7. Falagas, M.E.; Bliziotis, I.A. Pandrug-resistant Gram-negative bacteria: The dawn of the post-antibiotic era? Int. J. Antimicrob. Agents 2007, 29, 630-636. [CrossRef] [PubMed]

8. Ventola, C.L. The Antibiotic Resistance Crisis: Part 1: Causes and Threats. Pharm. Ther. 2015, 40, $277-283$.

9. Delcour, A.H. Outer Membrane Permeability and Antibiotic Resistance. Biochim. Biophys. Acta 2009, 1794, 808-816. [CrossRef] [PubMed]

10. Mishra, A.; Batra, S. Thiourea and guanidine derivatives as antimalarial and antimicrobial agents. Curr. Top. Med. Chem. 2013, 13, 2011-2025. [CrossRef] [PubMed]

11. Saczewski, F.; Balewski, Ł. Biological activities of guanidine compounds, 2008-2012 update. Expert Opin. Ther. Pat. 2013, 23, 965-995. [CrossRef] [PubMed]

12. Saeed, A.; Rafique, H.; Hameed, A.; Rasheed, S. Synthesis and antibacterial activity of some new 1-aroyl-3-(substituted-2-benzothiazolyl)thioureas. Pharm. Chem. J. 2008, 42, 191. [CrossRef]

13. Cunha, S.; Macedo, F.C.; Costa, G.A.N.; Rodrigues, M.T.; Verde, R.B.V.; de Souza Neta, L.C.; Vencato, I.; Lariucci, C.; Sá, F.P. Antimicrobial Activity and Structural Study of Disubstituted Thiourea Derivatives. Monatsh. Chem. 2007, 138, 511-516. [CrossRef]

14. Saeed, A.; Shaheen, U.; Hameed, A.; Naqvi, S.Z.H. Synthesis, characterization and antimicrobial activity of some new 1-(fluorobenzoyl)-3-(fluorophenyl)thioureas. J. Fluor. Chem. 2009, 130, 1028-1034. [CrossRef]

15. Andreev, K.; Bianchi, C.; Laursen, J.S.; Citterio, L.; Hein-Kristensen, L.; Gram, L.; Kuzmenko, I.; Olsen, C.A.; Gidalevitz, D. Guanidino Groups Greatly Enhance the Action of Antimicrobial Peptidomimetics against Bacterial Cytoplasmic Membranes. Biochim. Biophys. Acta 2014, 1838, 2492-2502. [CrossRef] [PubMed]

16. Bhat, M.; Belagali, S.L. Guanidinyl benzothiazole derivatives: Synthesis and structure activity relationship studies of a novel series of potential antimicrobial and antioxidants. Res. Chem. Intermediat. 2016, 42, 6195-6208. [CrossRef]

17. Böhm, H.-J.; Banner, D.; Bendels, S.; Kansy, M.; Kuhn, B.; Müller, K.; Obst-Sander, U.; Stahl, M. Fluorine in Medicinal Chemistry. Chem. Biol. Chem. 2004, 5, 637-643. [CrossRef] [PubMed]

18. Filler, R.; Saha, R. Fluorine in medicinal chemistry: A century of progress and a 60-year retrospective of selected highlights. Future Med. Chem. 2009, 1, 777-791. [CrossRef] [PubMed]

19. Deep, A.; Jain, S.; Sharma, P.C.; Mittal, S.K.; Phogat, P.; Malhotra, M. Synthesis, characterization and antimicrobial evaluation of 2,5-disubstituted-4-thiazolidinone derivatives. Arab. J. Chem. 2014, 7, 287-291. [CrossRef]

20. Krishnanjaneyulu, I.S.; Saravanan, G.; Vamsi, J.; Supriya, P.; Bhavana, J.U.; Sunil Kumar, M.V. Synthesis, characterization and antimicrobial activity of some novel benzimidazole derivatives. J. Adv. Pharm. Technol. Res. 2014, 5, 21-27. [CrossRef] [PubMed]

21. Stefanska, J.; Nowicka, G.; Struga, M.; Szulczyk, D.; Koziol, A.E.; Augustynowicz-Kopec, E.; Napiorkowska, A.; Bielenica, A.; Filipowski, W.; Filipowska, A.; et al. Antimicrobial and anti-biofilm activity of thiourea derivatives incorporating a 2-aminothiazole scaffold. Chem. Pharm. Bull. 2015, 63, 225-236. [CrossRef] [PubMed]

22. Douglass, I.B.; Dains, F.B. Some Derivatives of Benzoyl and Furoyl Isothiocyanates and their Use in Synthesizing Heterocyclic Compounds. J. Am. Chem. Soc. 1934, 56, 719-721. [CrossRef]

23. Saeed, A.; Flörke, U.; Erben, M.F. The role of substituents in the molecular and crystal structure of 1-(adamantane-1-carbonyl)-3-(mono)- and 3,3-(di) substituted thioureas. J. Mol. Struct. 2014, 1065-1066, 150-159. [CrossRef]

24. Saeed, A.; Erben, M.F.; Bolte, M. Synthesis, structural and vibrational properties of 1-(adamantane-1-carbonyl)-3-halophenyl thioureas. Spectrochim. Acta A Mol. Biomol. Spectrosc. 2013, 102, 408-413. [CrossRef] [PubMed] 
25. Mohamed, S.K.; Jaber, A.A.; Saeed, S.; Ahmad, K.S.; Wong, W.T. 1-(Adamantan-1-ylcarbon-yl)-3-(2,6-difluoro-4-hy-droxy-phen-yl)thio-urea. Acta Crystallogr. Sect. E. Struct. Rep. Online 2012, 68, o1597. [CrossRef] [PubMed]

26. Cunha, S.; Costa, M.s.B.; Napolitano, H.B.; Lariucci, C.; Vencato, I. Study of N-benzoyl-activation in the $\mathrm{HgCl} 2$-promoted guanylation reaction of thioureas. Synthesis and structural analysis of N-benzoyl-guanidines. Tetrahedron 2001, 57, 1671-1675. [CrossRef]

27. Gueirard, P.; Guiso, N. Virulence of Bordetella bronchiseptica: Role of adenylate cyclase-hemolysin. Infect. Immun. 1993, 61, 4072-4078. [PubMed]

28. Martina, P.; Leguizamon, M.; Prieto, C.I.; Sousa, S.A.; Montanaro, P.; Draghi, W.O.; Stammler, M.; Bettiol, M.; de Carvalho, C.; Palau, J.; et al. Burkholderia puraquae sp. nov., a novel species of the Burkholderia cepacia complex isolated from hospital settings and agricultural soils. Int. J. Syst. Evol. Microbiol. 2017, 68, 14-20. [CrossRef] [PubMed]

29. Martina, P.; Bettiol, M.; Vescina, C.; Montanaro, P.; Mannino, M.C.; Prieto, C.I.; Vay, C.; Naumann, D.; Schmitt, J.; Yantorno, O.; et al. Genetic Diversity of Burkholderia contaminans Isolates from Cystic Fibrosis Patients in Argentina. J. Clin. Microbiol. 2013, 51, 339-344. [CrossRef] [PubMed]

30. Baldwin, A.; Mahenthiralingam, E.; Thickett, K.M.; Honeybourne, D.; Maiden, M.C.; Govan, J.R.; Speert, D.P.; Lipuma, J.J.; Vandamme, P.; Dowson, C.G. Multilocus sequence typing scheme that provides both species and strain differentiation for the Burkholderia cepacia complex. J. Clin. Microbiol. 2005, 43, 4665-4673. [CrossRef] [PubMed]

31. CLSI Clinical and Laboratory Standards Institute. Methods for Dilution Antimicrobial Susceptibility Tests for Bacteria that Grow Aerobically; Approved Standard M07-A9, 9th ed.; CLSI: Wayne, PA, USA, 2012.

32. Isenberg, H.D. Synergism Testing: Broth Microdilution Checkerboard and Broth Macrodilution Methods. In Clinical Microbiology Procedures Handbook; American Society of Microbiology: Washington, DC, USA, 1992; pp. 1-28.

33. Berenbaum, M.C. Correlations between methods for measurement of synergy. J. Infect. Dis. 1980, 142, 476-480. [CrossRef] [PubMed]

34. Forcet, C.; Ye, X.; Granger, L.; Corset, V.; Shin, H.; Bredesen, D.E.; Mehlen, P. The dependence receptor DCC (deleted in colorectal cancer) defines an alternative mechanism for caspase activation. Proc. Natl. Acad. Sci. USA 2001, 98, 3416-3421. [CrossRef] [PubMed]

35. Orme, I. Search for new drugs for treatment of tuberculosis. Antimicrob. Agents Chemother. 2001, 45, 1943-1946. [PubMed]

36. Orzeszko, A.; Kaminska, B.; Starosciak, B.J. Synthesis and antimicrobial activity of new adamantane derivatives III. Farmaco 2002, 57, 619-624. [CrossRef]

37. Orzeszko, B.; Fedorynski, M.; Laudy, A.E.; Starosciak, B.J.; Orzeszko, A. Synthesis and antibacterial activity of 5-adamantan-1-yl-methyl analogues of trimethoprim. Acta Pol. Pharm. 2006, 63, 374-377. [PubMed]

38. Orzeszko, A.; Kaminska, B.; Orzeszko, G.; Starosciak, B.J. Synthesis and antimicrobial activity of new adamantane derivatives II. Farmaco 2000, 55, 619-623. [CrossRef]

39. Orzeszko, A.; Gralewska, R.; Starosciak, B.J.; Kazimierczuk, Z. Synthesis and antimicrobial activity of new adamantane derivatives I. Acta Biochim. Pol. 2000, 47, 87-94. [CrossRef]

40. Al-Abdullah, E.S.; Al-Tuwaijri, H.M.; Hassan, H.M.; Al-Alshaikh, M.A.; Habib, E.E.; El-Emam, A.A. Synthesis, Antimicrobial and Hypoglycemic Activities of Novel N-(1-Adamantyl)carbothioamide Derivatives. Molecules 2015, 20, 8125-8143. [CrossRef] [PubMed]

41. Levallet, C.; Lerpiniere, J.; Ko, S.Y. The $\mathrm{HgCl}_{2}$-promoted guanylation reaction: The scope and limitations. Tetrahedron 1997, 53, 5291-5304. [CrossRef]

42. Saeed, A.; Erben, M.F.; Abbas, N.; Flörke, U. Synthesis, crystal X-ray diffraction structure, vibrational properties and quantum chemical calculations on 1-(4-(4-Fluorobenzamido)phenyl)-3-(4-fluorobenzoyl)thiourea. J. Mol. Struct. 2010, 984, 240-245. [CrossRef]

43. Saeed, A.; Erben, M.F.; Shaheen, U.; Flörke, U. Synthesis, structural and vibrational properties of 1-(4-Fluorobenzoyl)-3-(isomeric fluorophenyl)thioureas. J. Mol. Struct. 2011, 1000, 49-57. [CrossRef]

44. Nzula, S.; Vandamme, P.; Govan, J.R.W. Influence of taxonomic status on the in vitro antimicrobial susceptibility of the Burkholderia cepacia complex. J. Antimicrob. Chemother. 2002, 50, 265-269. [CrossRef] [PubMed] 
45. Stover, C.K.; Pham, X.Q.; Erwin, A.L.; Mizoguchi, S.D.; Warrener, P.; Hickey, M.J.; Brinkman, F.S.; Hufnagle, W.O.; Kowalik, D.J.; Lagrou, M.; et al. Complete genome sequence of Pseudomonas aeruginosa PAO1, an opportunistic pathogen. Nature 2000, 406, 959-964. [CrossRef] [PubMed]

46. Vincent, J.L. Nosocomial infections in adult intensive-care units. Lancet 2003, 361, 2068-2077. [CrossRef]

47. Al-Khodor, S.; Marshall-Batty, K.; Nair, V.; Ding, L.; Greenberg, D.E.; Fraser, I.D. Burkholderia cenocepacia $\mathrm{J} 2315$ escapes to the cytosol and actively subverts autophagy in human macrophages. Cell. Microbiol. 2014, 16, 378-395. [CrossRef] [PubMed]

48. Chmiel, J.F.; Aksamit, T.R.; Chotirmall, S.H.; Dasenbrook, E.C.; Elborn, J.S.; LiPuma, J.J.; Ranganathan, S.C.; Waters, V.J.; Ratjen, F.A. Antibiotic Management of Lung Infections in Cystic Fibrosis. I. The Microbiome, Methicillin-Resistant Staphylococcus aureus, Gram-Negative Bacteria, and Multiple Infections. Ann. Am. Thorac. Soc. 2014, 11, 1120-1129.

49. Pick, N.; Cameron, S.; Arad, D.; Av-Gay, Y. Screening of Compounds Toxicity against Human Monocytic cell line-THP-1 by Flow Cytometry. Biol. Proced. Online 2004, 6, 220-225. [CrossRef] [PubMed]

50. Fröhlich, E. Toxicity of orally inhaled drug formulations at the alveolar barrier: Parameters for initial biological screening. Drug Deliv. 2017, 24, 891-905. [CrossRef] [PubMed]

51. George, A.M.; Jones, P.M.; Middleton, P.G. Cystic fibrosis infections: Treatment strategies and prospects. FEMS Microbiol. Lett. 2009, 300, 153-164. [CrossRef] [PubMed]

52. Gibson, R.L.; Burns, J.L.; Ramsey, B.W. Pathophysiology and Management of Pulmonary Infections in Cystic Fibrosis. Am. J. Respir. Crit. Care Med. 2003, 168, 918-951. [CrossRef] [PubMed]

53. Blazquez, J.; Couce, A.; Rodriguez-Beltran, J.; Rodriguez-Rojas, A. Antimicrobials as promoters of genetic variation. Curr. Opin. Microbiol. 2012, 15, 561-569. [CrossRef] [PubMed]

54. Leitao, J.H.; Sousa, S.A.; Cunha, M.V.; Salgado, M.J.; Melo-Cristino, J.; Barreto, M.C.; Sa-Correia, I. Variation of the antimicrobial susceptibility profiles of Burkholderia cepacia complex clonal isolates obtained from chronically infected cystic fibrosis patients: A five-year survey in the major Portuguese treatment center. Eur. J. Clin. Microbiol. Infect. Dis. 2008, 27, 1101-1111. [CrossRef] [PubMed]

55. Martina, P.; Feliziani, S.; Juan, C.; Bettiol, M.; Gatti, B.; Yantorno, O.; Smania, A.M.; Oliver, A.; Bosch, A. Hypermutation in Burkholderia cepacia complex is mediated by DNA mismatch repair inactivation and is highly prevalent in cystic fibrosis chronic respiratory infection. Int. J. Med. Microbiol. 2014, 304, 1182-1191. [CrossRef] [PubMed]

56. Chernish, R.N.; Aaron, S.D. Approach to resistant gram-negative bacterial pulmonary infections in patients with cystic fibrosis. Curr. Opin. Pulm. Med. 2003, 9, 509-515. [CrossRef] [PubMed]

57. Quon, B.S.; Aitken, M.L. Cystic Fibrosis: What to Expect now in the Early Adult Years. Paediatr. Respir. Rev. 2012, 13, 206-214. [CrossRef] [PubMed]

58. Lipuma, J.J. The changing microbial epidemiology in cystic fibrosis. Clin. Microbiol. Rev. 2010, 23, $299-323$. [CrossRef] [PubMed]

59. Parkins, M.D.; Floto, R.A. Emerging bacterial pathogens and changing concepts of bacterial pathogenesis in cystic fibrosis. J. Cyst. Fibros. 2015, 14, 293-304. [CrossRef] [PubMed]

60. Raczyńska, E.D.; Cyrański, M.K.; Gutowski, M.; Rak, J.; Gal, J.F.; Maria, P.C.; Darowska, M.; Duczmal, K. Consequences of proton transfer in guanidine. J. Phys. Org. Chem. 2003, 16, 91-106. [CrossRef]

61. Pfeffer, F.; Henderson, L.; Li, J.; Nation, R. Dioxolane Norbornane/Norbornene Compounds Suitable as Antimicrobial Agents to Treat Bacterial Infections. Patent WO2010099573A1, 10 September 2010.

(C) 2018 by the authors. Licensee MDPI, Basel, Switzerland. This article is an open access article distributed under the terms and conditions of the Creative Commons Attribution (CC BY) license (http://creativecommons.org/licenses/by/4.0/). 